Physical Chemistry: An Advanced Treatise. Vol. VIA: Kinetics of Gas Reactions. Edited by William Jost. Pp. $x x+507$. (Academic: New York and London, November 1974.) \$43.00; $£ 20.25$.

THE editors of this series note in the Foreword that, because of the tremendous expansion in the development of techniques and principles of physical chemistry in recent years, most physical chemists "find it difficult to maintain an understanding of the entire field". This surely understates the problem, and I must admit, albeit sadly, that I find this difficulty even when restricted to my own chosen field of physical chemistry-kinetics-let alone the "entire field".

An advanced treatise should assume that the reader has at least a first degree level of knowledge, and this is certainly true of this volume. Indeed in some cases rather more is assumed of the reader.

The first chapter, on formal kinetics, by W. Jost makes an excellent start with a concise but clear survey of classical reaction kinetics, and leads on to a consideration of steady states, stability and, finally such topics as oscillating reactions; these latter topics have attracted much attention in the past decade. The next, short chapter, by C. F. Curtiss-A Survey of Kinetic Theory - is, in my opinion, too limited in scope and is at a level which surely assumes too much of a graduate student. It does not fit in happily with the rest of the volume. In the third chapter by H. Eyring and S. H. Lin, on potential energy surfaces, an account of the valence bond method is given and then surfaces for $\mathrm{H}+\mathrm{H}_{2}, \mathrm{Cl}+\mathrm{Cl}_{2}$ and $\mathrm{K}+\mathrm{NaCl}$ are considered in some detail. A final section on orbital symmetry in reaction kinetics does not blend well with the rest of the chapter and deserves a chapter of its own.

Chapter 4 is again short-E. Nikitin on the Theory of Energy Transfer in Molecular Collisions. The translation, though clear, is a little stilted and the treatment is too condensed; few readers will find it an easy introduction to this important topic. The final chapters constitute more than half this volume. Chapter 5 by J. P. Toennies is on molecular beam scattering experiments and considers elastic, inelastic and reactive collisions. This is a field that is progressing rapidly and this chapter presents a balanced and clear account of it. Chapter 6 by J. C. Polanyi and J. L. Schreiber on the dynamics of bimolecular reactions must, of necessity, cover some of the same ground as the earlier chapters. Such duplication as there is, however, is not serious, and the viewpoint is somewhat different. Again, this chapter transitions". With that chapter one presents a clear account which most should also consider the following degraduate students interested in kinetics tailed treatment of the statistical theory will find valuable.

The chapter headings to the comof reactions. Similarly, the presentation panion volume (VIB) on gas kinetics which regards chemical reactions as suggest that the two will fit together diffusion processes of representative well. It is, however, sad to note how points in phase space from regions many important areas of gas kinctics corresponding to reactants to regions will not be covered at all.

This book can be recommended for libraries, but at $£ 20.25$ it is hardly cheap and is unlikely to find itself on the shelves of all those 'physical chemists' for whom it was, no doubt, intended.

H. M. Frey

\section{Gas works}

Theory of Elementary Atomic and Molecular Processes in Gases. By E. E. Nikitin. Translated by M. J. Kearsley. Pp. xiii+472. (Clarendon: Oxford; Oxford University Press: London, August 1974.) £14.75.

THE author of this book is one of the world's leading authorities on the theory of rate processes, who has extensive and detailed research experience in many branches of the subject. This translation from the Russian version originally published in 1970 is, therefore, more than welcome. The emphasis rests on the theory of processes relevant to the chemical reactions discussed. The main body of the book makes reference to the literature published before the end of 1966, with an added appendix that includes brief reference to developments in the two or three ycars following that date.

Professor Nikitin presents a very wide, well integrated view of the subject as a whole, and thus the material in the various chapters of the book is well organised, with chapters following one another in a smooth fashion. The first chapter on the transition state method is presented in a very formal manner, going far beyond the statistical mechanical treatment normally found in standard books on kinetics. The following chapter, dealing with the transfer of translational motion to other forms of energy, especially to vibration, for which there is a large body of experimental data, and also to electronic energy in both atoms and diatomic molecules, is extensive and highly authoritative.

The chapter formally entitled "Unimolecular Reactions" may seem to be relatively limited, considering the scope of the subject, but it is a tight and formal approach. It follows well the chapter concerned with vibrational energy transfer and includes a section on surface crossing and "non-adiabatic shelves. corresponding to products, is also highly relevant to the considerations of unimolecular reactions given in earlier chapters.

The dissociation of diatomic molecules and the reverse process of atomic terms of the types of results obtained from different theories than in terms of detailed developments of the leading theories. The appeal to experiment is very limited but the chapter is rich in the physical principles at issue in such processes. The basis of semi-empirical presented with particular clarity, and leads in due course to the more recent, $a b$ initio calculations of Bruner and Conroy for the $\mathrm{H}_{3}$ system.

Direct reactions and the dynamics of exchange reactions, of particular relevance to processes studied in molecular beams, are also dealt with fully. The appendix, clearly written after the main body of the book, is a useful brief resumé of the objectives of theories of chemical reactions. As the book is designed for post graduate chemists and physicists, the appendix could usefully be read first, especially as there is no general introduction.

Since the completion of the book, there has been a major development in the experimental study of many fundamental aspects of atomic reactions. Work on the collisional behaviour of atoms in specific electronic states, and velopment of a framework within which context the relationship between electronic structure and atomic reactivity can be understood, comprise much of this development. These areas of research occupy little space, however, and it is to be hoped that the next edition will contain much more of that type of work, including Professor Nikitin's own theoretical researches.

For research workers concerned either with the theory or experiment of fundamental rate processes in gases, access to this book is barely a matter of choice but rather of basic necessity. Occasionally, the price of a book must be judged not only in relation to its size but to the quality of its content. This is such a book; it is hard to imagine a library without it, and it is to be hoped that research workers will consider that a classic work of this kind merits a place on their personal bookrecombination are dealt with more in methods for bimolecular reactions is various studies aimed towards the de- 